\title{
A Review of Metallic Fractal Aggregates
}

\author{
Rodolfo J. Slobodrian, Claude Rioux, Michel Piché \\ Département de Physique, de Génie physique et D’optique, Université Laval, Québec, Canada \\ E-mail: rjslobodrian@hotmail.com \\ Received September 25, 2011; revised November 2, 2011; accepted November 18, 2011
}

\begin{abstract}
Metals are the main components of the Earth's mass and are characterized by high thermal and electrical conductivity as well as high reflectivity of electromagnetic fields. Finely divided metals are efficient catalysers and this indicates the relevance of surfaces when their ratio to volume becomes large. This is a characteristic of fractal aggregates and their constituent monomers (spheroidal or other) in the micrometer to nanometer scales. Exotic fern shaped aggregates are also produced. All aggregates exhibit large ratios of surface to volume. Condensation of metallic vapours allows to obtain particle sizes much smaller than those obtained via grinding techniques and far superior in purity. Exotic alloys of non miscible metals have been obtained at the micrometer scale. Thermal and laser evaporation methods of metals followed by condensation are described. Low gravity aggregation experiments were also carried out on aircraft in parabolic flight.
\end{abstract}

Keywords: Fractals, Monomers, Aggregates, Metals, Alloys

\section{Introduction}

Fractals are ubiquitous in our universe including both inanimate and living matter, at all scales, from subatomic to cosmic dimensions [1]. Physical fractals are deemed to be a fourth state of matter, additional to the usual solid, liquid and gaseous states. Fractal is a word coined by Benoit Mandelbrot as well as the expressions fractal geometry and fractal dimension [2]. The foundations are to be found in the development of the concepts of topological and metric spaces, dimension and measure, laid by $19^{\text {th }}$ and $20^{\text {th }}$ century mathematicians, particularly Cavalieri, Cantor, Lebesgue, Hausdorff, Banach, Borel, Kuratowski and Kolmogorov [3], essential for the understanding of the generalisation leading to non-integer dimensions (fractal dimensions). In 1982 Mandelbrot defined a fractal as a set for which the Hausdorff-Besicovitch dimension strictly exceeds the topological dimension. This abstract definition was replaced by Mandelbrot in 1986 introducing the concept of self-similarity: Fractal is a shape (set) made by parts similar to the whole in some way [4].This definition implies scale invariance of parts of a set. Physical fractals have a finite range of scale invariance whereas mathematical fractals have infinite range. Physical fractals are characterized by randomness and exhibit frequently dendritic structures.

\section{Fractal Dimension}

Integer dimensions are assigned axiomatically: 0 for a point, 1 for a line, 2 for a surface, etc., based on the assumption of continuity. However, for example a subset of the points on a line in correspondence with the rational numbers should reflect a dimension below 1 . This is based on the idea of measure of a set with adequate covering units: sticks, plates, cubes or balls, etc. For instance to measure the area of the floor of a room it is possible to use surface elements of variable linear dimension $\lambda$ and cover the floor to any given accuracy. The capacity (concept due to Kolmogorov) of the covering element can be expressed as

$$
\mathrm{C}=\mathrm{K} \lambda^{\mathrm{n}}
$$

For squares $K=1$, for disks $K=\pi, n=2$ in both cases. For cubes $K=1, n=3$, for spheres (balls) $K=4 / 3 \pi$ and $\mathrm{n}=3$. For the covering of the floor the area $\mathrm{A}$ is then given by

$$
\mathrm{A}_{\lambda}=\mathrm{N}_{\lambda} \mathrm{C}
$$

If the surface to be measured is not dense the covering elements can be expressed as

$$
\mathrm{C}=\mathrm{K} \lambda_{\lambda}^{\mathrm{D}}
$$

where $\mathrm{D}_{\lambda}<2$ accounting for the non-dense surface and 
substituting the topological dimension. Thus now the area can be expressed as

$$
\mathrm{A}=\mathrm{NK} \lambda_{\lambda}^{\mathrm{D}}
$$

With the heuristic choice $A=1 \mathrm{~K}=1$, the fractal dimension $\mathrm{D}$ is given by $\lambda$

$$
\mathrm{D}=\lim \left[\ln \mathrm{N}_{\mathrm{D}_{\lambda}} \cdot \ln (1 / \lambda)\right]
$$

Mutatis mutandis the expression is valid for systems embedded in 3-dimensional space.

Figure 1 visualizes the method to determine the fractal dimension by the slope of the graph.

\section{Fractal Characteristics}

A specific nomenclature has been developed to designate traits of fractals. This is demonstrated in Figure 2. Branches are called dendrites (name borrowed from Greek: trees and neurons), deep cavities are Fjords (from Norway's landscapes), and individual elements of the aggregate are known as monomers, derived from chemical polymers, which are similar to aggregates.

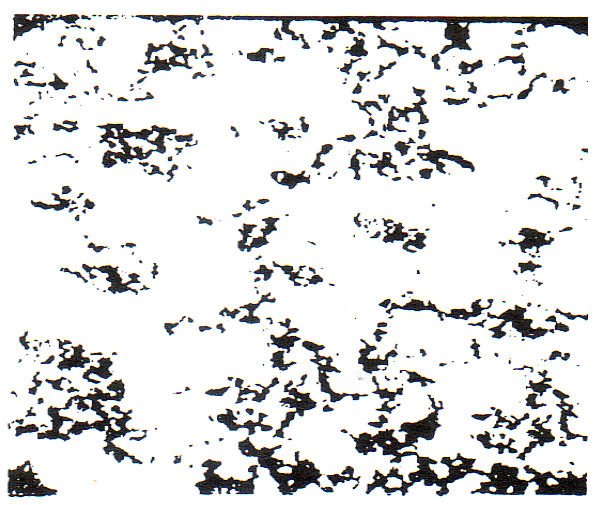

(a)

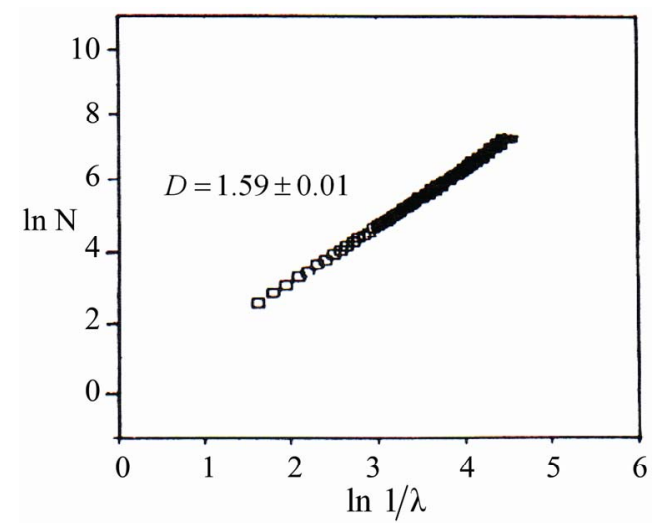

(b)

Figure 1. (a) 2-Dimensional fractal; (b) A graph of its fractal dimension.

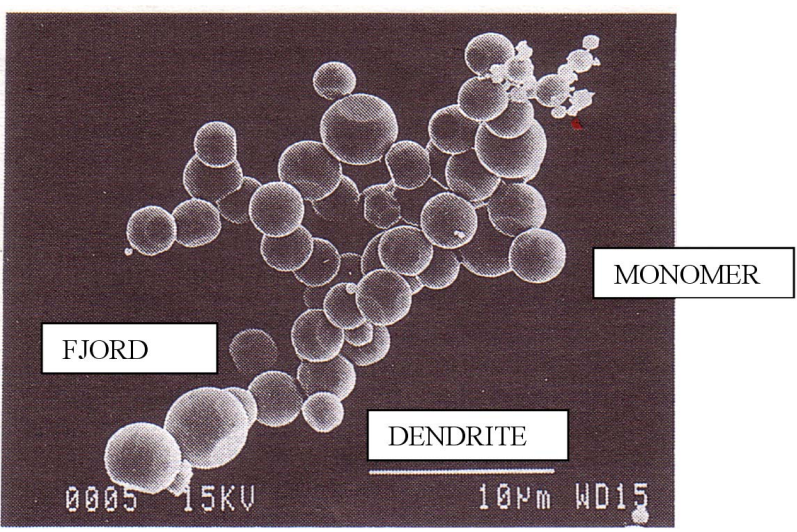

Figure 2. SEM image of a 3-dimensional Zn aggregate. Notice at the upper right a secondary aggregate with monomers down to the nanometer scale. It is a beautiful example of self-similarity in aggregates.

The density of fractals is related to their fractal dimension because the latter reflects the occupancy of space by particles. The mass $\mathrm{M}$ of a fractal of dimension $\mathrm{D}$ enclosed in a cube of side $\mathrm{L}$ is thus proportional to $\mathrm{L}^{\mathrm{D}}(\mathrm{D}<$ 3). Therefore the matter density in the cube is

$$
\rho \sim \mathrm{L}^{(\mathrm{D}-3)}
$$

Consequently it goes to zero when $\mathrm{L}=>\infty$. The aggregate grows tenuous and in normal gravity tends to collapse. Low gravity environments (real or simulated) are necessary for a proper study of the growth and properties of fractal aggregates.

\section{Reduced Gravity Environments}

Free fall of a system in vacuum provides a means of eliminating gravity. This technique has given rise to drop towers and provides for several seconds of near zero g. Aircraft in parabolic flight can mock-up conditions near free fall for periods of tens of seconds with jitters above the level of g from drop towers. Space shuttles, asteroids, the forthcoming aerospace plane and the International Space Station (stationary orbits) can provide long periods of near zero gravity. Space probes far from gravitational fields may provide unsurpassed periods of picogravities but may require elaborate chains of signal transmission stations [5].

However, a simulation of low gravity conditions can be accomplished in ground based laboratories by flotation of particles in an inert gas atmosphere. Such medium is also required in condensation experiments in order to reduce the velocities of evaporated atoms to thermal levels. Otherwise, in vacuum, these atoms would be projected onto the walls of the experimental enclosures. Metallic elements would coat the surfaces and no 3-d aggregation could 
occur. The preferred gas of experiments referred to here was argon, although helium and krypton were also used occasionally. It is reasonable to assume that the supporting gas does not interact in any significant way with the evaporated atoms and provides only flotation to the aggregating particles. Ground based experiments have been carried out with an evaporator using ohmic heating of the metals equipped with a gas handling system. A schematic of the evaporation-condensation system is shown in Figure 3. Anodized tungsten crucibles were used to evaporate the metals. During the evaporation convection played a relevant role and a smoke-like column would ascend above the crucible, a saddle shaped vortex was also formed, as depicted in Figure 4.

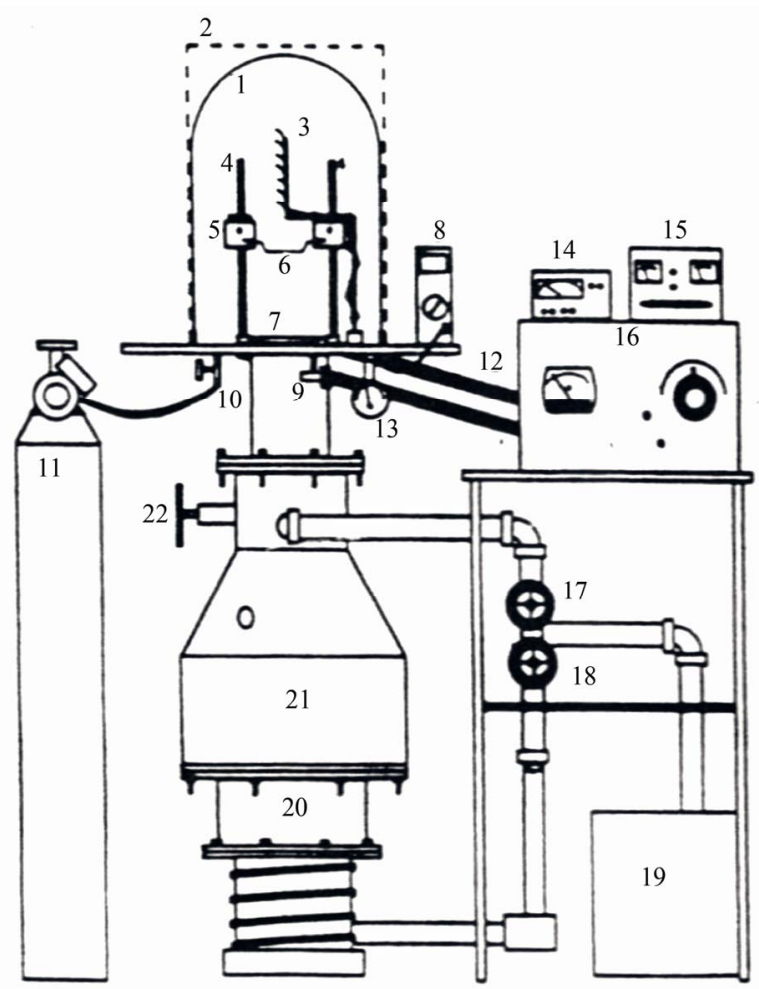

Figure 3.

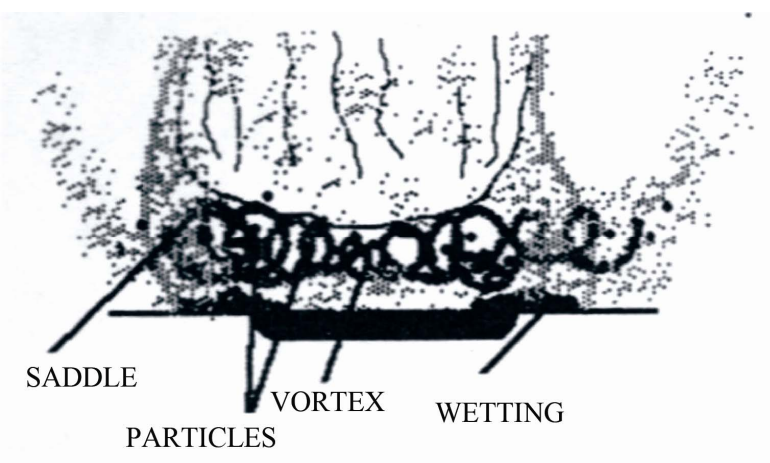

Figure 4. The crucible in operation.
Seventeen metals were evaporated and their condensation produced aggregates of varied shapes. Aggregates with typical monomers were analyzed by scattering of He-Ne laser radiation (Figure 5) to determine the fractal dimension D from the angular distribution of the intensity I(q), where $q$ is the modulus of the difference of the initial and final wave vectors (Figure 6).This is a non-destructive indirect measurement method [6] .

A direct measurement of the fractal dimension of a physical aggregate may become possible with the forthcoming ESA-ICAPS facility on the ISS, with a fast holographic record of the growth of aggregates, providing the coordinates of the monomers or particles aggregating $[7,8]$, but it is presently geared to the study of powders only. It may be extended to vapors in some future developments.

Several examples of SEM images of metallic fractal aggreates are shown in Figures 7 and 8.

The fern like aggregates shown in Figures $\mathbf{9}$ and $\mathbf{1 0}$

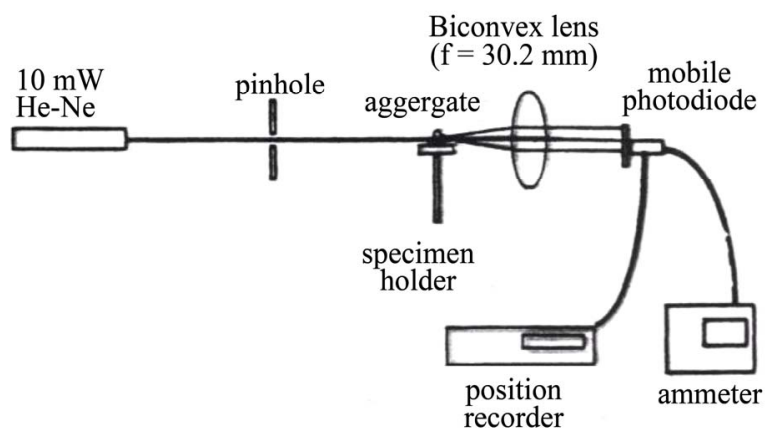

Figure 5. Laser scattering apparatus schematic.

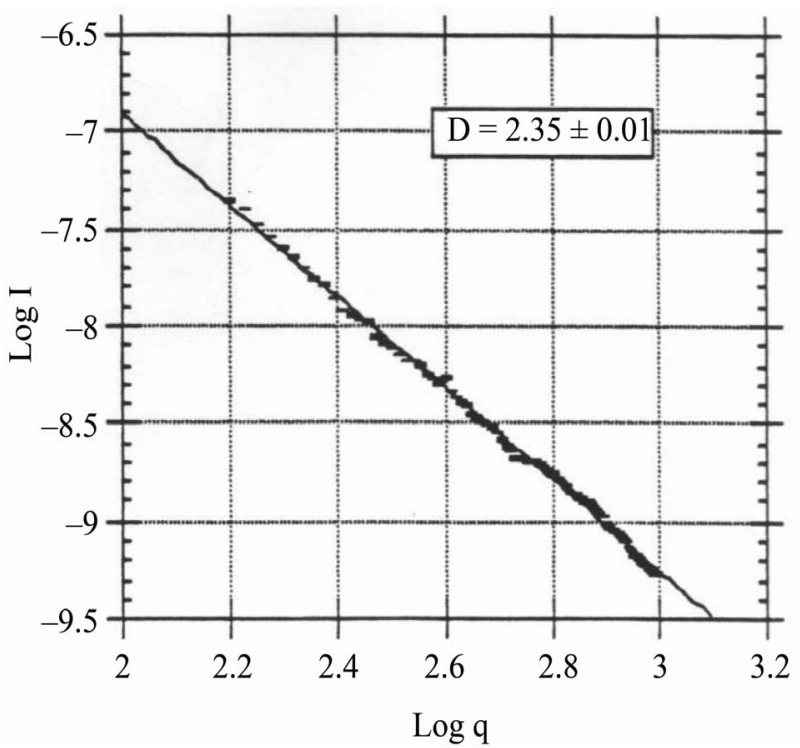

Figure 6. Example of a measurement of $D$ of a 3-d Zn aggregate. 
below exhibit branches of dendrites demonstrating a sequence of self similar structures at gradually diminishing scales. This is a striking demonstration of the scale invariance of fractal aggregates.

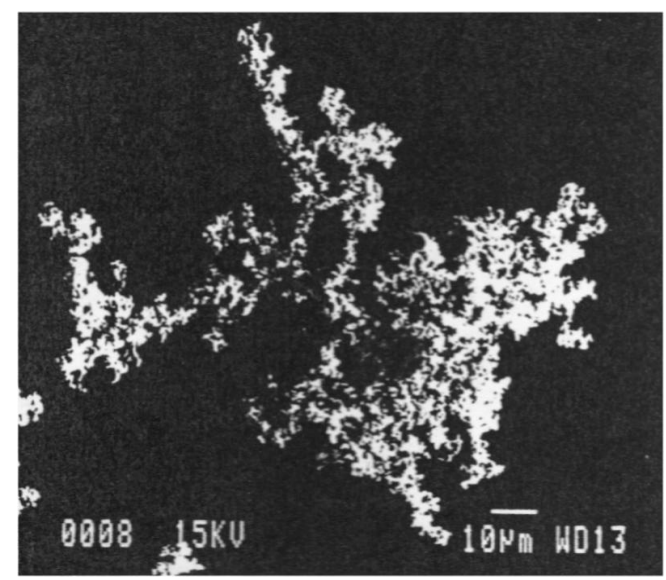

Figure 7. Aggregate of Mn.

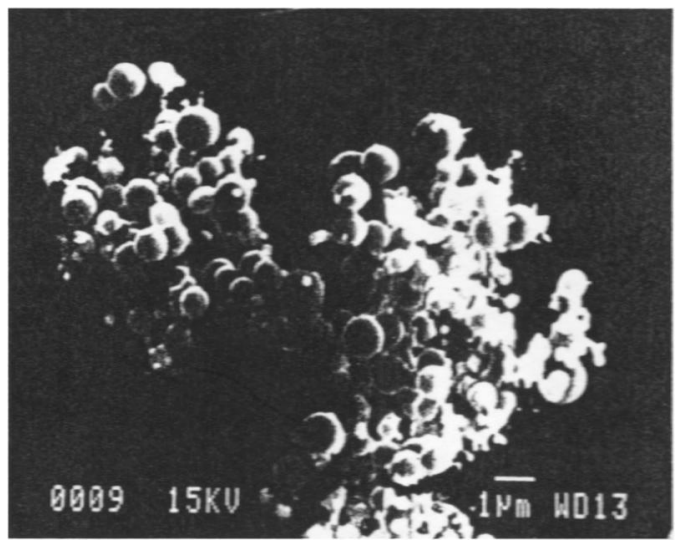

Figure 8. Enlargement of a part of Figure 7.

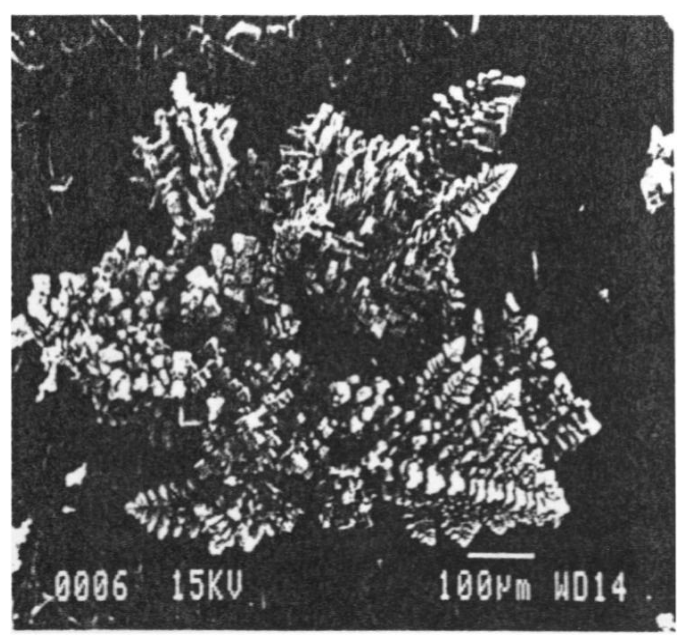

Figure 9. Aggregate of $\mathrm{Cr}$ with a fern like pattern, the monomers are tetrahedral.

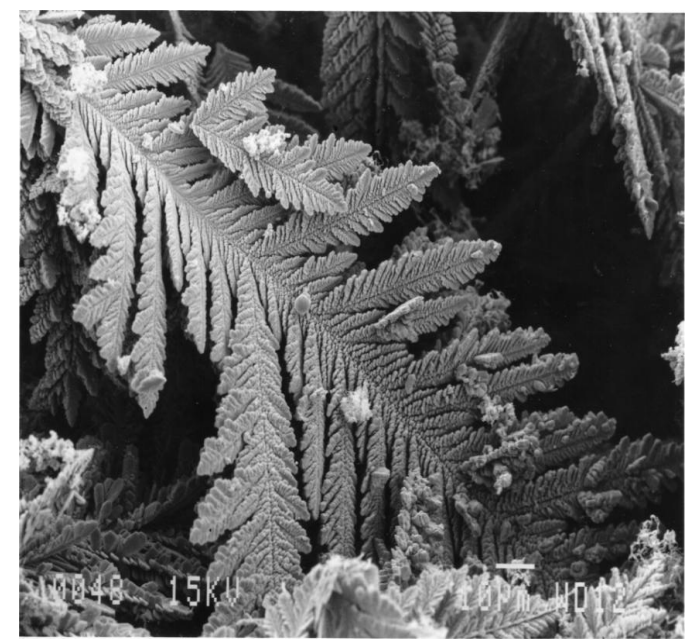

Figure 10. Fern like aggregate of $\mathrm{Zn}$.

\section{Experiments in Low Gravity of Parabolic Flights}

Ground based experiments of evaporated metals in a gaseous medium suffer from motions induced by thermal gradients which limit the growth of the aggregates as well as introduce distortions. Experiments were carried out on aircraft of NASA, ESA and NRC-Canada. One early experiment carried out with powders allowed to establish the relevance of electrostatic interactions in aggregations [9]. See Figure 11 below.
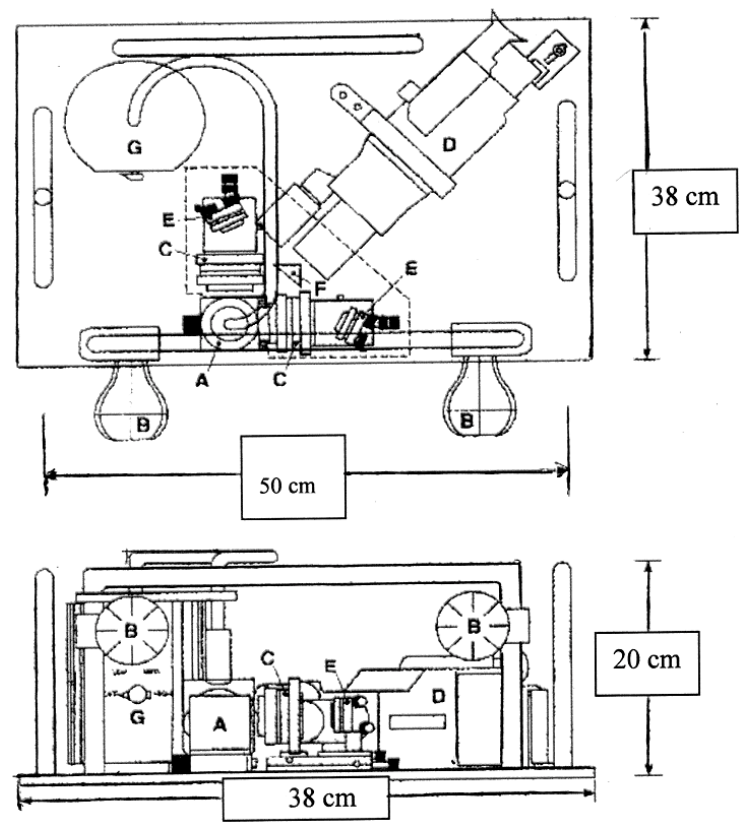

Figure 11. Apparatus used for experiments of NASA's KC-135 aircraft, horizontal and vertical views. A: Aggregation cell; B: Particle activation bellows; C: Lenses; D: Recording camera; E: Mirrors; F: Prism. 
The apparatus records on a single frame of the camera two perpendicular images of the aggregations within the cell, thus assuring a perfect synchronization of the images. This allows to reconstruct the 3 -d paths of aggregating poarticles.

Experiments with evaporated metals were carried out with the apparatus of Figure 12.

Experiments were carried out in parabolic flight on NASA's aircraft, KC-135, DC 9 and NRC-Canada Fal- con. The most remarkable aggregate obtained was of $\mathrm{Zn}$ with gigantic proportions shown on Figure 13. It is an example of multiple fractal aggregation (MFA) [10], such that several aggregates are condensed simultaneously from the metallic vapors. Most simulation programmes of physical aggregation deal with a single system.

We have developed a computer programme to generate three dimensional multiple fractal aggregation (MFA) of spheroidal monomers.

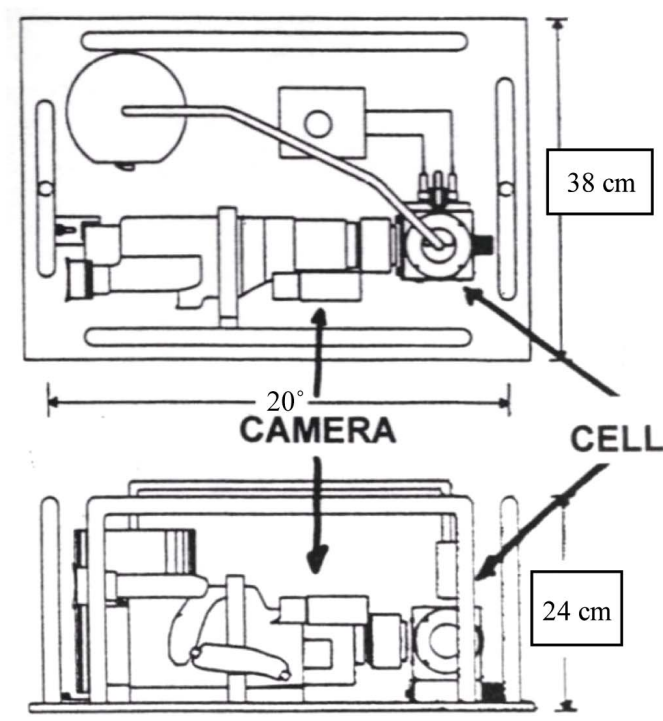

(a)
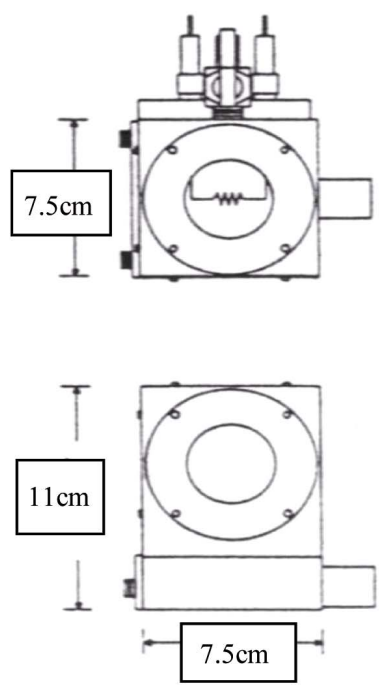

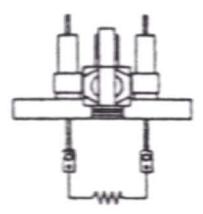

FILAMENT

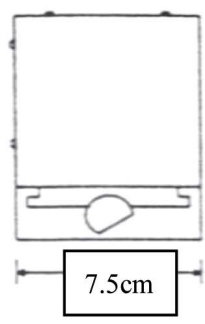

(b)

Figure 12. (a) Horizontal and vertical views of the apparatus; (b) Detail of the cubic experimental cell. The evaporation proceeds via ohmic heating.

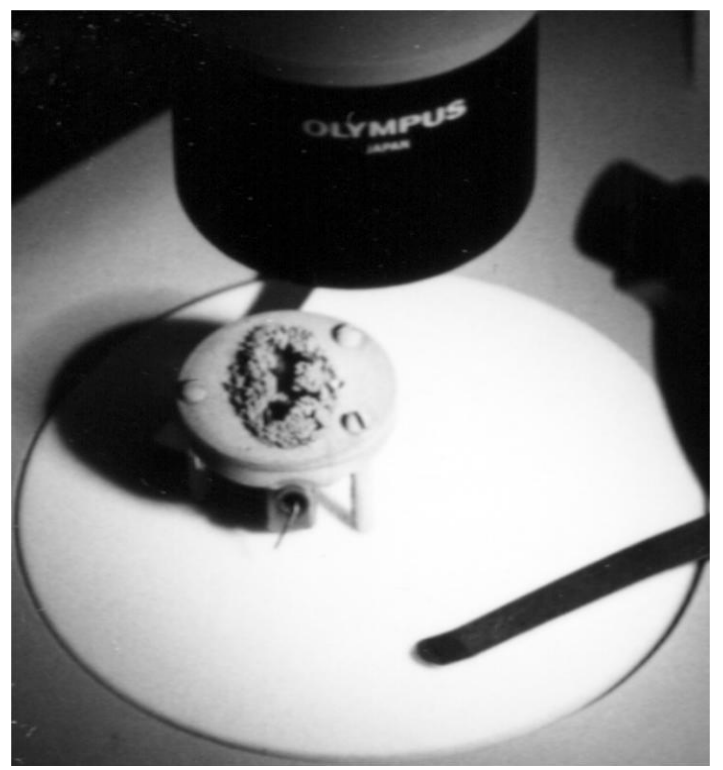

(a)

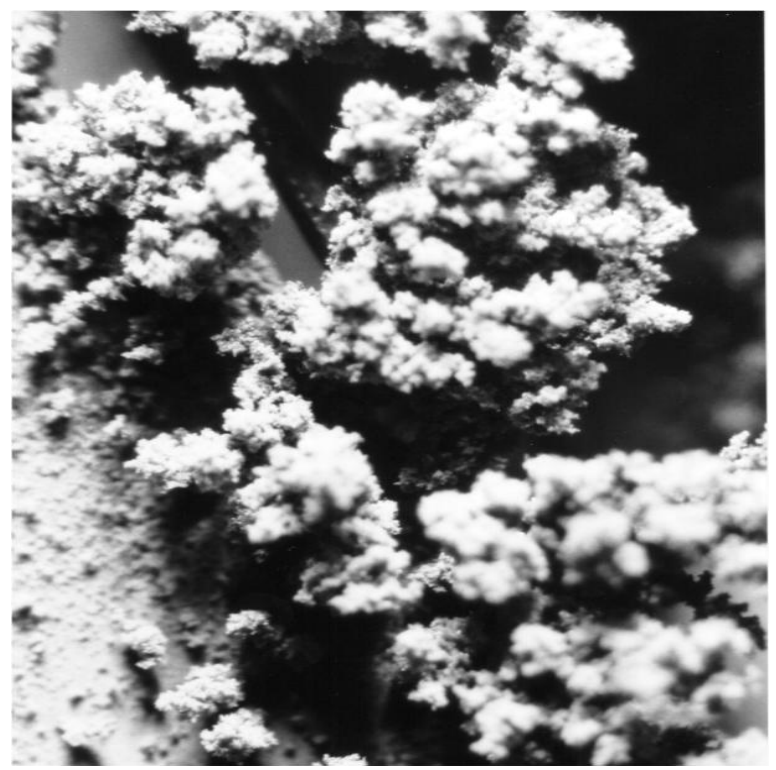

(b)

Figure 13. (a) View of a big Zn aggregate; (b) Optical microscope view os part of the Zn aggregate at left. 


\section{Exotic Alloy of $\mathrm{Al}$ and $\mathrm{Ag}$}

A result crowning the experiments using ohmic evaporation is shown in Figures $\mathbf{1 5}$ and $\mathbf{1 6}$ the exotic alloy of aluminium and silver obtained. The aggregates shown are also of the type MFA of Figure 14.

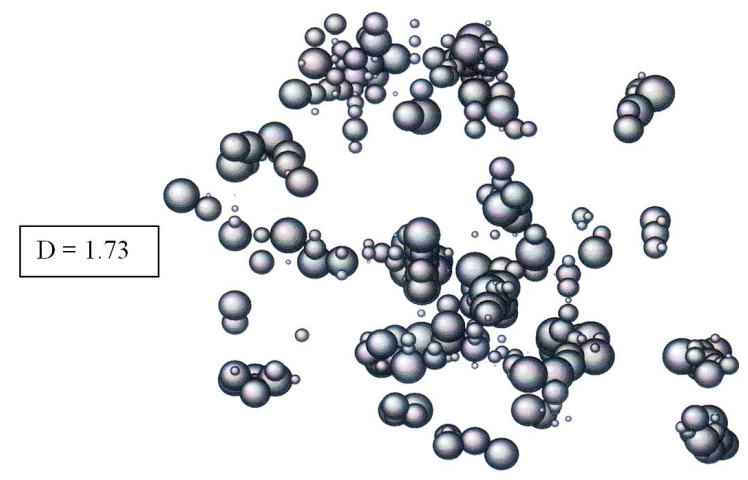

Figure 14. Example of MFA simulation. D is the multifractal dimension obtained via the box counting method.

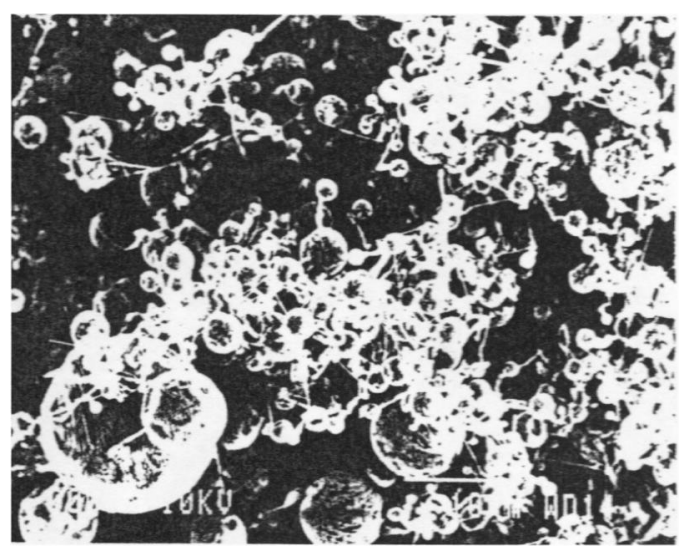

Figure 15. SEM image of an alloy of Al-Ag.

Series II Laval University

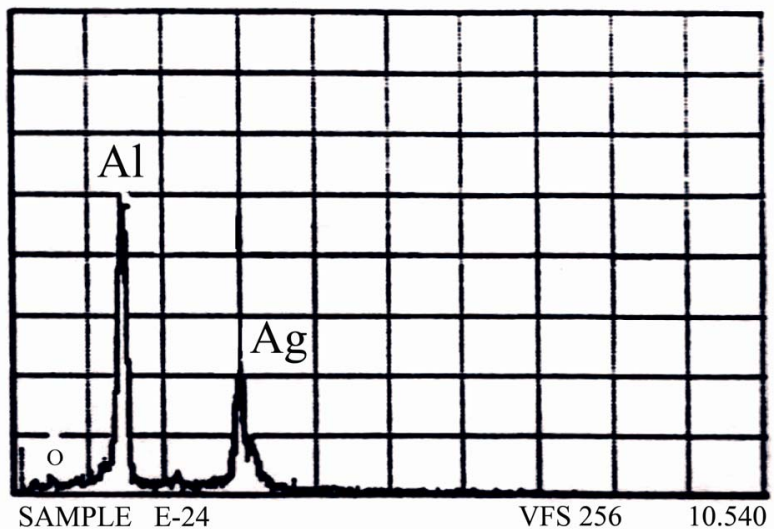

Figure 16. Mass spectrum of Figure 15 confirming the composition.

\section{Laser Vapourization and Condensation of Metals}

Lasers are far superior to ohmic heating to produce vapours of high purity, but the required installation is considerably more complex (shown in Figure 17).

A basic laser (see Table 1) set-up is shown in Figure 18 for vaporization of targets.

An aggregate generated via laser vaporisation is shown in Figure 19.

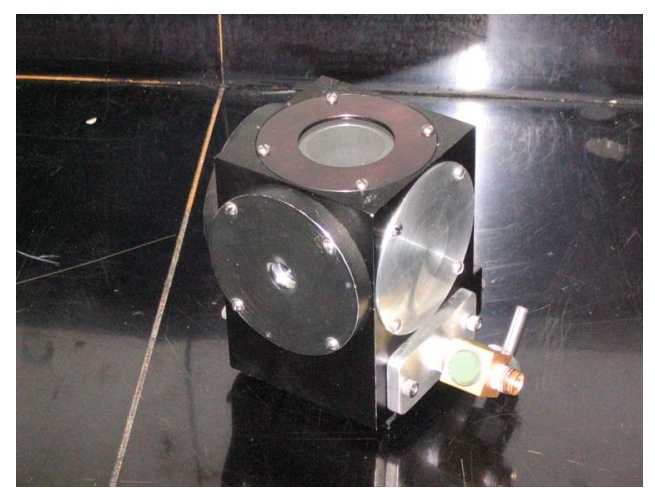

Figure 17. Photo of the experimental cube.

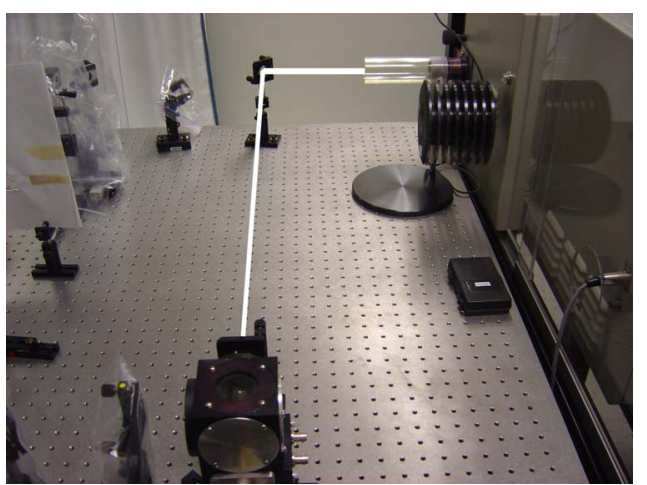

Figure 18. The target in the experimental cube is at the bottom left.

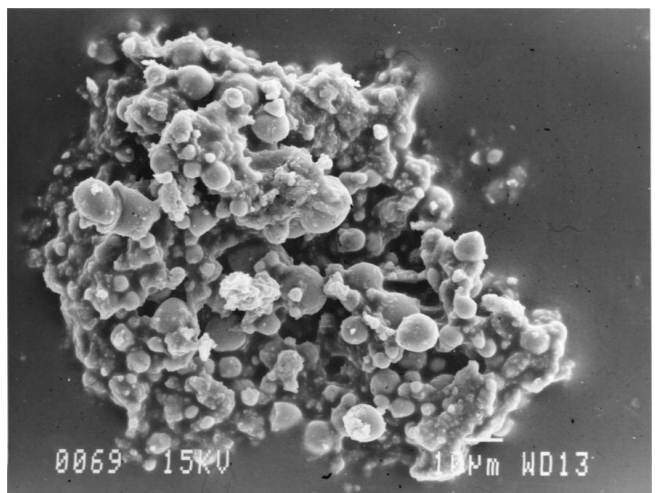

Figure 19. A condensed aggregate from laser generated vapours. 
Table 1. Laser characteristics.

\begin{tabular}{ccc} 
Parameters & Nominal values & Values used \\
Wavelength (nm) & 248 & 248 \\
Maximum energy per pulse (mJ) & 450 & 120 à 240 \\
Mean energy maximum (W) & 80 & 1 à 2,5 \\
Repetition rate (pps) & 200 & 10 \\
Length of pulse (ns) & 12 à 20 & 12 à 20 \\
Size of the beam (mm) & $8-12 \times 25$ & $8-12 \times 25$ \\
Divergence of the beam (mrad) & $1 \times 3$ & $1 \times 3$ \\
\hline
\end{tabular}

\section{Alloy of Aluminium and Tungsten}

A laser set-up was prepared in order to split the beam in two and convey them at $90^{\circ}$ onto targets of two metals in order to produce intersecting beams of $\mathrm{Al}$ and $\mathrm{W}$ thus allowing the simultaneous condensation into monomers. The beam was split using a prism as shown in Figure 20.

The full beam paths are illustrated in Figure 21.

The coliding beams generated by laser interaction are shown in Figure 22.

The mass spectrum analysis shown in Figure 23 indicates the success generating the Al-W alloy.

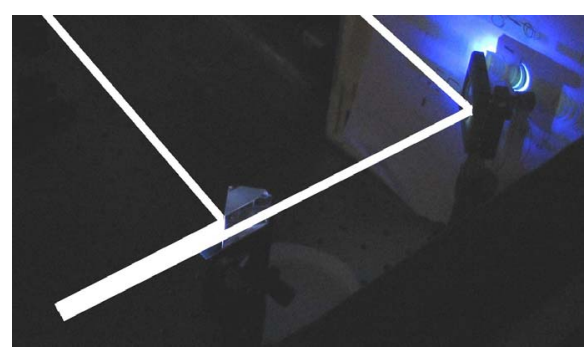

Figure 20. Photo showing the splitting of the laser beam into components.

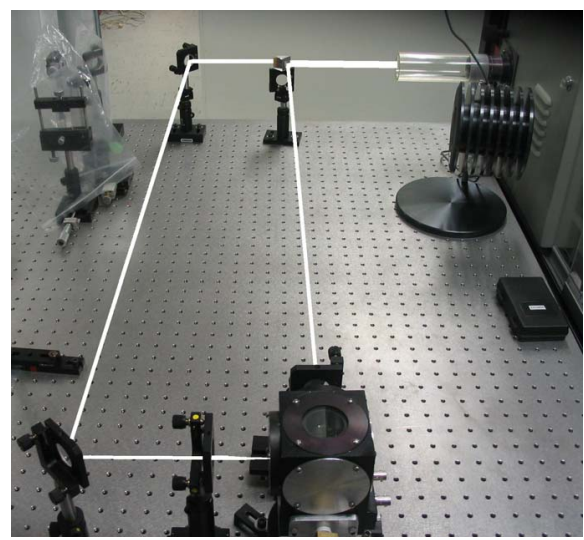

Figure 21. The targets are placed in the box at the lower centre.

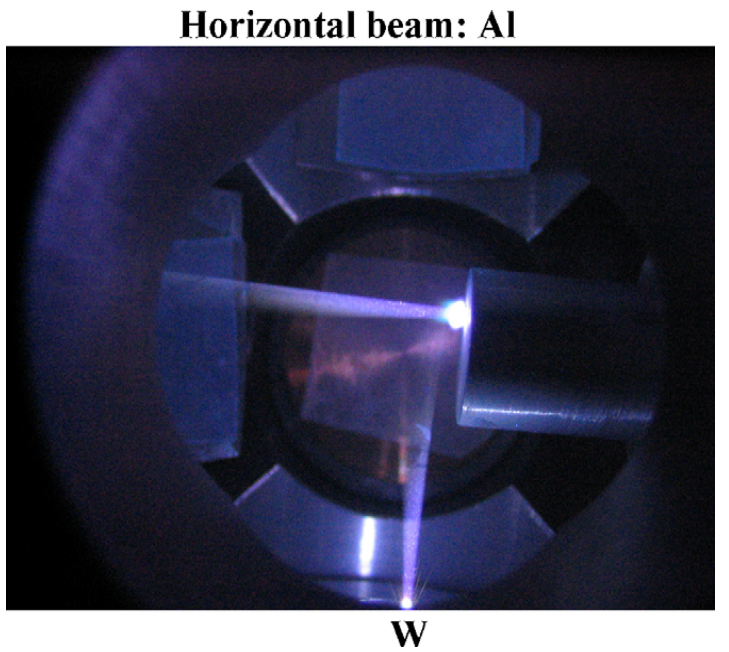

Figure 22. Photograph showing the colliding beams inside the target chamber.

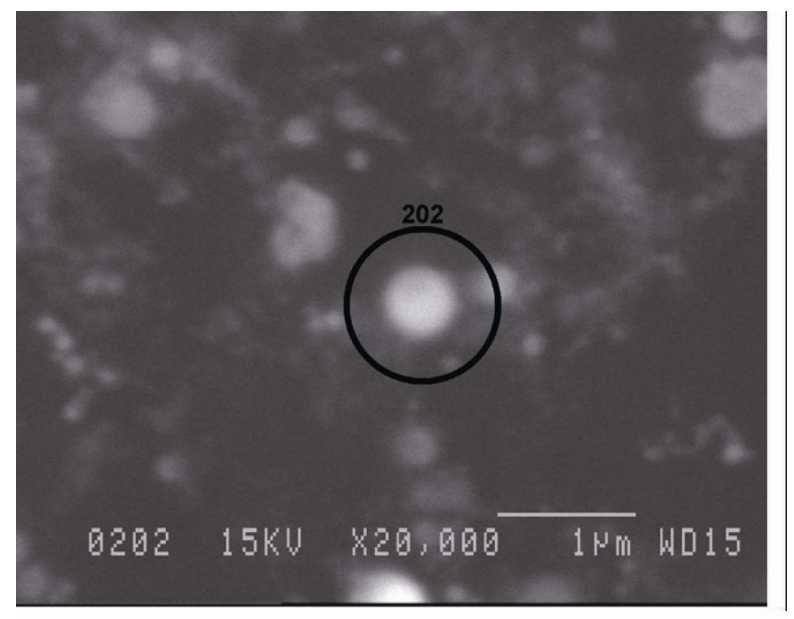

Series II Laval University $\quad \mathrm{Al}-\mathrm{W}$ aggregates

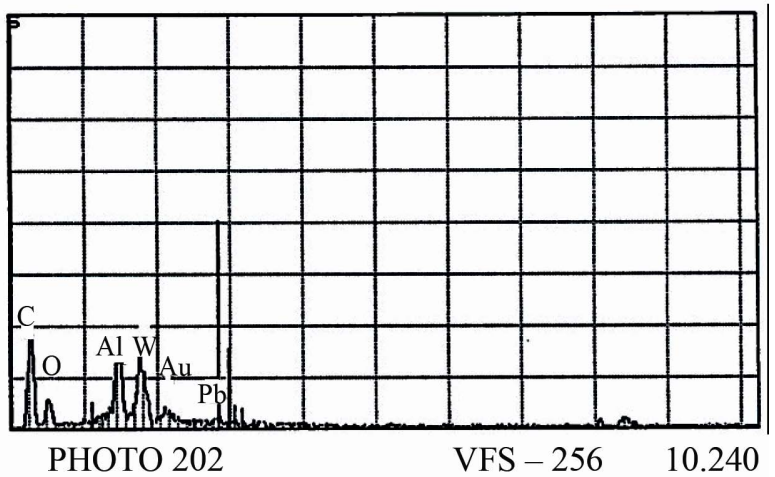

Figure 23. Upper: SEM image of monomers the circle indicates a monomer whose mass spectrum is shown (lower diagram). Alumnium is indicated by $\mathrm{Al}$, tungsten by $\mathrm{W}$.

\section{Concluding Remarks}

Fractal aggregates of metals at the micro- and nano-me- 
ter scales provide physical systems with exceptional.

The successful result of alloying two immiscible metals opens up the production of exotic alloys with several components and exceptional properties [11]. properties due to the dominance of surfaces over volumes. They also constitute a bridge between the realm of quantum phenomena and macroscopic classical physics phenomena. This region is poorly known and worthy of fundamental research because Bohr's correspondence principle may not be adequate to comprehend the transition from atomic systems to macroscopic matter [12]. Further work on this important aspectt is foreseen.

\section{Acknowledgements}

This review reflects the toil and enthousiasm of a sizeable number of researchers and it would be too long to enumerate. The support of technical shops of the university was essential to this work carried out mostly with special equipment constructed locally. Financial support was provided intermittently by the Canadian and European Space Agencies (CSA and ESA) it is thanked kindly.

\section{References}

[1] R. J. Slobodrian, "Fractal Cosmogony: Similarity of the Early Universe to Microscopic Fractal Aggregates,” Chaos, Solitons \& Fractals, Vol. 23, No. 3, 2005, pp. 727-729.

[2] B. Mandelbrot, "Les Objets Fractals, Forme, Hasard et Dimension,” 3rd Edition, Flammarion, Paris, 1989.

[3] G. A. Edgar, Ed. “Classics on Fractals,” Addison-Wesley
Publishing Company, Boston, 1993.

[4] J. Feder, “Fractals,” Plenum Press, New York, 1988.

[5] R. J. Slobodrian and C. Rioux, "Test of Modified Newtonian Dynamics in Picogravity: The Dark Matter Alternative Solution," Japan Society of Microgravity Applications, Vol. 25, No. 3, 2008, pp. 243-246.

[6] P. Deladurantaye, et al., "Effect of Gravity on the Growth of Fractal Aggregates," Chaos, Solitons \& Fractals, Vol. 8, No. 10, 1997, pp. 1693-1709. doi:10.1016/S0960-0779(96)00133-6

[7] J. Blum, et al., "Dust from Space," Europhysics News, Vol. 39, No. 3, 2008, pp. 27-29. doi:10.1051/epn:2008303

[8] A. Orr, "The IPE Facility in the ISS," 38th COSPAR Scientific Assembly, Bremen, 15-18 July 2010, p. 293.

[9] C. Rioux, L. Potvin and R. J. Slobodrian, "Particle-Particle Aggregation with $1 / \mathrm{r}^{2}$ Forces in Reduced Gravity Environments,” Physical Review E, Vol. 52, No. 2, 1995, pp. 2099-2101. doi:10.1103/PhysRevE.52.2099

[10] M. Laliberté, et al., "A Clue for Unusual Metallic Aggregates with Monomers in the Nanometer Scale," Chaos, Solitons \& Fractals, Vol. 22, No. 4, 2004, pp. 935-938. doi:10.1016/j.chaos.2004.03.014

[11] J.-C. Leclerc, Ph. Binette, M. Piché, N. McCarthy, C. Rioux and R. J. Slobodrian, "Alloys of Aluminium and Tungsten in the Micrometer Scale," Journal of Alloys and Compounds, Vol. 452, No. 2, 2008, pp. L1-L4. doi:10.1016/j.jallcom.2006.11.070

[12] R. J. Slobodrian, "Quantum Aspects of Fractal Structures and Monomers," Chaos, Solitons \& Fractals, Vol. 14, No. 6, 2002, pp. 817-822. 